\title{
Intrinsic Vector-Valued Symmetric Form for Simple Mechanical Control Systems in the Nonzero Velocity Setting
}

\author{
Jason Nightingale, Richard Hind and Bill Goodwine
}

\begin{abstract}
We obtain an intrinsic vector-valued symmetric bilinear form that can be associate with an underactuated simple mechanical control system. We determine properties of the form which serve as necessary conditions for driving underactuated simple mechanical control systems to rest. We also determine properties of the form that serve as sufficient conditions for driving a simple mechanical systems underactuated by one control to an $\varepsilon$-neighborhood of rest from an arbitrary initial configuration and velocity. These conditions are computable and coordinate invariant. We focus on the case where the symmetric form is real-valued and indefinite on the entire configuration manifold. Our technical results give rise to a nonlinear control law that drives these systems to an $\varepsilon$-neighborhood of rest given an arbitrary initial configuration, velocity and $\varepsilon>0$.
\end{abstract}

\section{INTRODUCTION}

\section{A. Background}

Mechanical systems form a large subset of nonlinear control systems which have numerous diverse and challenging applications. Such areas include autonomous aerospace and marine vehicles, robotics and automation, and multi-body systems. Moreover, the areas of overlap between mechanics and control possess mathematical elegance that makes them appealing to study independent of applications. There currently exists a limited understanding of the set of reachable states from nonzero velocity states for simple mechanical control systems [1]. The underlying mathematical structure of mechanical systems is that of second-order dynamics on the tangent bundle of the configuration manifold where the state of the system is defined by a configuration and velocity. Controllability results that are limited to zero velocity states do not provide an adequate characterization of the behavior of mechanical systems and limit the extension of local accessibility and controllability results to the larger class of hybrid nonlinear mechanical systems [2].

Underactuated mechanical control systems are interesting to study both from a theoretical and a practical point of view. Theoretically, they are a challenge to control due to nonzero drift. Practically, they appear in numerous applications resulting from design choices motivated by cost reductions or in some cases they are the result of a failure in fully actuated mechanical systems.

This work was supported by the Center for Applied Mathematics at the University of Notre Dame

J. Nightingale is a graduate student Mechanical Engineering and Mathematics, University of Notre Dame, Notre Dame, Indiana 46556 USA jnightin@nd.edu

R. Hind is with the Department of Mathematics, University of Notre Dame, Notre Dame, Indiana 46556 USA rhindend. edu

B. Goodwine is with the Department of Aerospace and Mechanical Engineering, University of Notre Dame, Notre Dame, Indiana 46556 USA jgoodwinend.edu
In general, the problem of determining controllability for underactuated nonlinear control systems is very difficult. Sussmann's work [3] on sufficient conditions for small-time local controllability forms the cornerstone of many existing analyses of mechanical control systems. Lewis and Murray [4] have extended the general results of Sussmann to simple mechanical systems in the zero velocity setting by taking advantage of the inherent structure in the governing equations of motion. The reachable set from zero initial velocity can be constructed using symmetric products and Lie brackets. It is well-known that each of these results are not feedback invariant. Consequently, several efforts have been made to obtain conditions in the zero velocity setting from properties of a certain intrinsic vector-valued quadratic form which does not depend upon the choice of basis for the input distribution [1], [5].

A slightly weaker notion of controllability for locomotive systems is referred to as fiber controllability [7]. Fiber controllability refers to the system's ability to control certain states with no concern for other. A nice example is the mathematical model of a swimming robot [9]. Cortes et al. [8] provide sufficient conditions local fiber controllability for mechanical systems with symmetries and constraints in the zero velocity setting.

\section{B. Statement of Contribution}

We seek to develop a geometric tool that can be used to characterize the set of reachable velocities. The basic strategy is to pick a specific target in the tangent bundle and develop necessary and sufficient conditions for reaching that target. Our target is the zero section of the tangent bundle or rest since the current controllability results for simple mechanical systems are restricted to zero initial velocity [6]. We ask the following two questions for simple mechanical control systems:

1) Starting from a given configuration and velocity, is it possible to reach rest?

2) If so, is it possible to design a nonlinear control law that will drive this system to rest?

We obtain computable results which are dependent upon coordinate invariant properties of an intrinsic vector-valued symmetric bilinear form that can be associated with a simple mechanical control system. Specifically, we provide necessary conditions for reaching rest from an arbitrary initial configuration and velocity (see Section III.C, Theorem 12). We also provide sufficient conditions and a nonlinear control law for driving a class of simple mechanical system underactuated by one control to an $\varepsilon$-neighborhood of rest from an 
arbitrary initial configuration and velocity (see Section III.C, Theorem 15).

Our unique approach is to use the governing equations of motion to partition or foliate the phase manifold. Similar to fiber controllability, an implicit notion throughout this paper is that of global foliation controllability. We develop a method to measure a simple mechanical control system's ability to move among leaves of the input foliation. Our analysis may also be extended to a larger class of nonlinear mechanical systems with isotropic dissipation [10] and constrained mechanical control systems [11]. These results are applied to the forced planar rigid body.

\section{GEOMETRIC MODEL}

\section{A. Simple Mechanical Control Systems}

We consider a simple mechanical control system which is comprised of an $n$-dimensional configuration manifold $M$; a Riemannian metric $\mathbb{G}$ which represents the kinetic energy; a $\mathbb{R}$-valued function $V$ on $M$ which represents the potential energy; $m$ linearly independent one forms $F^{1}, \ldots, F^{m}$ on $M$ which represents the input forces; and $U=\mathbb{R}^{m}$ which represents the set of inputs. We do not require the set of inputs to be a subset of $\mathbb{R}^{m}$. This allows use to focus on the geometric properties of our system that inhibit or allow motion in the foliation as opposed to a limitation on the set of inputs. We represent the input forces as one forms and use the associated dual vector fields $Y_{a}=\mathbb{G}^{\sharp}\left(F^{a}\right), a=1, \ldots, m$ in our computations. Formally, we denote the control system by the tuple $\Sigma=\{M, \mathbb{G}, \mathscr{Y}, V, U\}$ where $\mathscr{Y}=\left\{Y_{a} \mid Y_{a}=\right.$ $\left.\mathbb{G}^{\sharp}\left(F^{a}\right) \forall a\right\}$ is the input distribution. Note we restrict our attention to control systems where the input forces are dependent upon configuration and independent of velocity and time. DoCarmo [12] provides an excellent introduction to Riemannian geometry. A thorough description of simple mechanical control systems is provided by Bullo and Lewis [6].

It is well known that the Lagrange-d'Alembert principle can be used to generate the equations of motion for a forced simple mechanical system in coordinate invariant form [13]. If we set the Lagrangian equal to the kinetic energy minus potential energy, then the equations are given by

$$
\nabla_{\dot{\gamma}(t)} \dot{\gamma}(t)=-\operatorname{grad} V(\gamma(t))+u^{a}(t) Y_{a}(\gamma(t))
$$

where $\nabla$ is the Levi-Civita connection corresponding to $\mathbb{G}$, $u$ is a map from $I \subset \mathbb{R} \mapsto \mathbb{R}^{M}, \gamma: I \rightarrow M$ is a curve on $M$ and $t \in I$. Therefore, a controlled trajectory for $\Sigma$ is taken to be the pair $(\gamma, u)$ where $\gamma$ and $u$ are defined on the same interval $I \subset \mathbb{R}$. Note the usual summation notation will be assumed over repeated indices throughout this paper.

The natural coordinates on $T M$ are denoted by $\left(\left(q^{1}, \ldots, q^{n}\right),\left(v^{1}, \ldots, v^{n}\right)\right)$ where $\left(v^{1}, \ldots, v^{n}\right)$ are the coefficients of a tangent vector given the usual basis $\left\{\frac{\partial}{\partial q^{1}}, \ldots, \frac{\partial}{\partial q^{n}}\right\}$. We will denote a point in $T M$ by $v_{q}$. We may lift the second-order differential equation defined by (1) to
$T M$. This gives rise to the following system of first-order differential equations on $T M$

$$
\begin{aligned}
& \frac{d q^{k}}{d t}=v^{k} \\
& \frac{d v^{k}}{d t}=-\Gamma_{i j}^{k} v^{i} v^{j}+u^{a} Y_{a}^{k}-\operatorname{grad} V^{k}
\end{aligned}
$$

where $\Gamma_{i j}^{k}$ is the usual Christoffel symbol and $i, j, k=1, \ldots, n$. Equation (2) is the local representation of the following vector field on $T M$

$$
\begin{aligned}
\dot{\xi}(t)= & Z(\xi(t))+u^{a}(t) Y_{a}^{\mathrm{vlft}}(\pi(\xi(t))) \\
& -\operatorname{grad} V^{\mathrm{vlft}}(\pi(\xi(t))) .
\end{aligned}
$$

where $\xi(t)$ is the total state, $Z$ is the geodesic spray, $\pi$ is the canonical projection $T M \mapsto M$, and $Y_{a}^{v l f t}, \operatorname{grad} V^{v l f t}$ are the vertical lifts of the vector fields $Y_{a}, \operatorname{grad} V$ on $M$. Recall that the vertical lift of a vector field $X$ at the point $v_{q}$ is denoted by $X_{v_{q}}^{\mathrm{vlft}}$ and is the tangent vector at $t=0$ to the curve $t \mapsto v+t X$.

A critical tool used to analyze distributions and mechanical control systems is the symmetric product. Given a pair of vector fields $X, Y$, their symmetric product is the vector field defined by

$$
\langle X: Y\rangle=\nabla_{X} Y+\nabla_{Y} X
$$

We denote the closure of the distribution $\mathscr{Y}$ with respect to the symmetric product by $\operatorname{Sym}^{\infty}(\mathscr{Y})$. Recall that a distribution $\mathscr{Y}$ is geodesically invariant if and only if it is closed with respect to the symmetric product [14].

The Lie derivative of a real-valued function $f$ on $M$ with respect to the vector field $X=X^{i} \frac{\partial}{\partial q^{i}}$ is defined in coordinates by

$$
L_{X}(f)=X^{i} \frac{\partial}{\partial q^{i}}(f) .
$$

\section{B. Forced Planar Rigid Body}

In this section we introduce the geometric model of the forced planar rigid body (Fig. 1).

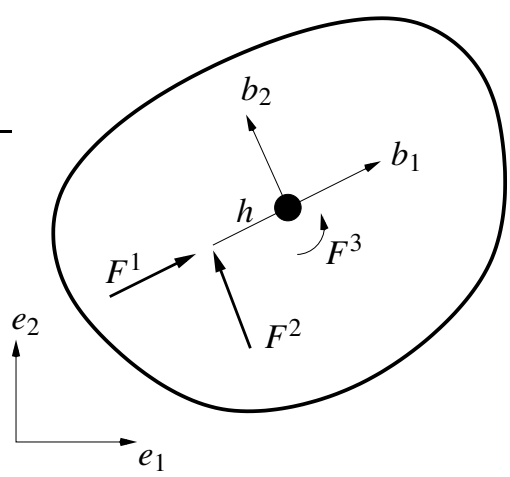

Fig. 1. Forced Planar Rigid Body

The configuration manifold for the system is the Lie group $S E(2)$ and the potential function is assumed to be identically zero. Let us use coordinates $(x, y, \theta)$ for the planar robot 
where $(x, y)$ describes the position of the center of mass and $\theta$ describes the orientation of the body frame $\left\{b_{1}, b_{2}\right\}$ with respect to the inertial frame $\left\{e_{1}, e_{2}\right\}$. In these coordinates, the Riemannian metric is given by

$$
\mathbb{G}=m d x \otimes d x+m d y \otimes d y+J d \theta \otimes d \theta,
$$

where $m$ is the mass of the body and $J$ is the moment of inertia about the center of mass. The inputs for this system consist of forces applied to an arbitrary point and a torque about the center of mass. We assume that the point of application of the force is a distance $h>0$ from the center of mass along the $b_{1}$ body-axis. Physically, the input force can be thought of as a variable-direction thruster on the body which can be resolve into components along the $b_{1}$ and $b_{2}$ directions. The control inputs are given by

$$
\begin{aligned}
& F^{1}=\cos \theta d x+\sin \theta d y, \\
& F^{2}=-\sin \theta d x+\cos \theta d y-h d \theta, \\
& F^{3}=d \theta .
\end{aligned}
$$

\section{GEOMETRIC ANALYSis}

\section{A. Construction}

In this section we expand upon and adapt the definition of an affine subbundle found in Hirschorn and Lewis [5]. We restrict our attention to configuration manifolds that admit a well defined global set of basis vector fields however our results generalize under appropriate conditions. Recall that an input distribution $\mathscr{Y}$ on $M$ is a subset $\mathscr{Y} \subset T M$ having the property that for each $q \in M$ there exists a family of vector fields $\left\{Y_{1}, \ldots, Y_{m}\right\}$ on $M$ so that for each $q \in M$ we have

$$
\mathscr{Y}_{q} \equiv \mathscr{Y} \cap T_{q} M=\operatorname{span}_{\mathbb{R}}\left\{Y_{1}(q), \ldots, Y_{m}(q)\right\} .
$$

We refer to the vector fields $\left\{Y_{1}, \ldots, Y_{m}\right\}$ as generators for $\mathscr{Y}$. Let $\mathscr{Y}^{\perp}$ denote the orthonormal frame $\left\{Y_{1}^{\perp}, \ldots, Y_{n-m}^{\perp}\right\}$ that generates the $\mathbb{G}$-orthogonal complement of the input distribution $\mathscr{Y}$. It is clear that $\left\{\mathscr{Y}_{q}, \mathscr{Y}_{q}^{\perp}\right\}$ form a basis for $T_{q} M$ at each $q \in M$. Note that $\mathscr{Y}=\left\{Y_{1}, \ldots, Y_{m}\right\}$ is a set of $m$ linearly independent vector fields while $\mathscr{Y}^{\perp}=\left\{Y_{1}^{\perp}, \ldots, Y_{n-m}^{\perp}\right\}$ is a set of $n-m$ orthonormal vector fields. This basis will be used to define an affine subbundle and construct an affine foliation of the tangent bundle.

An affine subbundle on $M$ is a subset $A \subset T M$ having the property that for each $q \in M$ there exists a family of vector fields $\left\{Y_{0}, \ldots, Y_{m}\right\}$ so that for each $q \in U$ we have

$$
\begin{aligned}
A_{q} \equiv & A \cap T_{q} M \\
= & \left\{Y_{0}(q)=Y_{1}^{\perp}(q)+\cdots+Y_{n-m}^{\perp}(q)\right\} \\
& +\operatorname{span}_{\mathbb{R}}\left\{Y_{1}(q), \ldots, Y_{m}(q)\right\} .
\end{aligned}
$$

An affine foliation, $\mathscr{A}$, on $T M$ is a collection of disjoint immersed affine subbundles of $T M$ whose disjoint union equals $T M$. Each connected affine subbundle $A$ is called an affine leaf of the affine foliation. Now let us apply this framework to a simple mechanical control system.
Definition 1 (Input Foliation): Let $(M, \mathbb{G}, V, \mathscr{Y}, U)$ be a simple mechanical control system with the input distribution $\mathscr{Y}$ generated by $\left\{Y_{1}, \ldots, Y_{m}\right\}$ and the corresponding $\mathbb{G}$ orthogonal distribution $\mathscr{Y}^{\perp}$ generated by $\left\{Y_{1}^{\perp}, \ldots, Y_{n-m}^{\perp}\right\}$. An input foliation $\mathscr{A} y$ is an affine foliation whose affine leaves are affine subbundles given by

$$
A_{s}(q)=\left\{v_{q} \in T M \mid\left\langle\left\langle Y^{\perp}, v_{q}\right\rangle\right\rangle=s, s \in \mathbb{R}^{n-m}\right\} .
$$

Remark 2: The input foliation is parametrized by $s \in$ $\mathbb{R}^{n-m}$. Note that when $s=0, A_{0}=\mathscr{Y}$ and $A_{0}(q)=\mathscr{Y}_{q}$ where $\mathscr{Y}$ is an immersed submanifold of $T M$ and $\mathscr{Y}_{q}$ is a linear subspace of $T_{q} M$. Thus, the input distribution $\mathscr{Y}$ is a single leaf of the affine foliation.

Now we introduce the notion of the generalized symmetric Christoffel symbols.

Definition 3: We define the generalized symmetric Christoffel symbols for $\nabla$ with respect to the basis of vector fields $\left\{X_{1}, \ldots, X_{n}\right\}$ on $M$ as the $n^{3}$ functions $\tilde{\Gamma}_{i j}^{k}: M \rightarrow \mathbb{R}$ defined by

$$
\frac{1}{2}\left\langle X_{i}: X_{j}\right\rangle=\tilde{\Gamma}_{i j}^{k} X_{k}
$$

Remark 4: If we let $X_{i}=\frac{\partial}{\partial q^{i}}$ then we recover the usual Christoffel symbols.

We may define the velocity vector $\dot{\gamma}(t)=\dot{\gamma}^{i}(t) \frac{\partial}{\partial q^{i}}$ of the curve $\gamma(t)$ in terms of the family of vector fields $\left\{\mathscr{Y}, \mathscr{Y}^{\perp}\right\}$. The new expression for $\dot{\gamma}(t)$ is in the form

$$
\dot{\gamma}(t)=w^{a}(t) Y_{a}(\gamma(t))+s^{b}(t) Y_{b}^{\perp}(\gamma(t))
$$

where $s^{b}(t)=\left\langle\left\langle\dot{\gamma}(t), Y_{b}^{\perp}\right\rangle\right\rangle_{\gamma(t)}$. We now provide a local expression for a measure of a simple mechanical control system's ability to move among the leaves of the input foliation $\mathscr{A}_{\mathscr{Y}}$.

Lemma 5: Let $(M, \mathbb{G}, V, \mathscr{Y}, U)$ be a simple mechanical control system with an input foliation $\mathscr{A}_{\mathscr{Y}}$ defined above. The following holds along the curve $\gamma(t)$ satisfying (1):

$$
\begin{aligned}
\frac{d}{d t} s^{b}(t)= & -\frac{1}{2} w^{a}(t) w^{p}(t)\left\langle\left\langle\left\langle Y_{a}: Y_{p}\right\rangle, Y_{b}^{\perp}\right\rangle\right\rangle \\
& -\frac{1}{2} w^{a}(t) s^{r}(t)\left\langle\left\langle\left\langle Y_{a}: Y_{r}^{\perp}\right\rangle, Y_{b}^{\perp}\right\rangle\right\rangle \\
& -\frac{1}{2} s^{r}(t) w^{p}(t)\left\langle\left\langle\left\langle Y_{r}^{\perp}: Y_{p}\right\rangle, Y_{b}^{\perp}\right\rangle\right\rangle \\
& -\frac{1}{2} s^{r}(t) s^{k}(t)\left\langle\left\langle\left\langle Y_{r}^{\perp}: Y_{k}^{\perp}\right\rangle, Y_{b}^{\perp}\right\rangle\right\rangle \\
& -\left\langle\left\langle\operatorname{grad} V, Y_{b}^{\perp}\right\rangle\right\rangle
\end{aligned}
$$

where $a, p \in\{1, \ldots, m\}, b, k, r \in\{1, \ldots, n-m\}$.

Proof: Recall from Definition 1 that

$$
s^{b}(t)=\left\langle\left\langle Y_{b}^{\perp}, \dot{\gamma}(t)\right\rangle\right\rangle
$$

holds along $\gamma(t)$. We could precede by substituting (4) into (6) and differentiating taking advantage of the compatibility associated with the Levi-Civita connection. Alternatively, we use the notion of a generalized symmetric Christoffel symbol. It follows from the construction of $\mathscr{Y}^{\perp}$ that the $b$ th 
component of $\tilde{\Gamma}_{i j}^{b}$ along the the orthonormal vector field $Y_{b}^{\perp}$ can be expressed as a projection using $\mathbb{G}$.

We observe that (5) is quadratic in the parameter $w(t)$. Now we relate an intrinsic vector-valued symmetric bilinear form to the measure derived in Lemma 5.

Definition 6: Let $(M, \mathbb{G}, V, \mathscr{Y}, U)$ be a simple mechanical control system with the input distribution $\mathscr{Y}$ generated by $\left\{Y_{1}, \ldots, Y_{m}\right\}$ and the corresponding $\mathbb{G}$-orthogonal distribution $\mathscr{Y}^{\perp}$ generated by $\left\{Y_{m+1}^{\perp}, \ldots, Y_{n}^{\perp}\right\}$. We define the intrinsic vector-valued symmetric bilinear form to be $B: \mathscr{Y}_{q} \times \mathscr{Y}_{q} \rightarrow$ $T_{q} M / \mathscr{Y}_{q}$ given in coordinates by

$$
B_{a p}^{b}=\frac{1}{2}\left\langle\left\langle\left\langle Y_{a}: Y_{p}\right\rangle, Y_{b}^{\perp}\right\rangle\right\rangle
$$

where $a, p \in\{1, \ldots, m\}, b \in\{1, \ldots, n-m\}$.

Remark 7: The intrinsic vector-valued symmetric bilinear form defined above tells us exactly how the velocity components $w$ parallel to the input forces influence the velocity components $s$ orthogonal to the input forces. The remainder of the paper will focus on characterizing computable, coordinate invariant properties of $B$.

Remark 8: If $\Sigma$ is underactuated by one control then $b=1$ and $B$ is a real-valued symmetric bilinear form.

\section{B. Control Definitions}

The following section contains several control definitions which are used in the statement of our main results.

Definition 9: We say that $\Sigma$ is $\varepsilon$-stabilizable to rest $(\varepsilon$ STR) if for any $\varepsilon>0$ there exists a piecewise continuous function $\tilde{u}: T M \rightarrow \mathbb{R}^{m}$ such that the solution to the initial value problem

$$
\dot{\xi}(t)=Z(\xi(t))+\tilde{u}^{a}(\xi(t)) Y_{a}^{v l f t}(\xi(t)), \quad \xi(0)=\left(q_{0}, v_{0}\right),
$$

satisfies $|v(T)|<\varepsilon$ for some $q \in M$ and finite $T$.

Definition 10: Given a $m \times m$ semi-definite real-valued symmetric bilinear form $B$ of $\operatorname{rank}(k)$ where $k \leq m$, we can always construct a $k \times k$ definite real-valued symmetric bilinear form $\tilde{B}$ by eliminating $m-k$ linearly dependent rows and columns. We say that $\tilde{B}$ is the minimal definite realvalued symmetric bilinear form on $M$.

Definition 11: Let $B$ be a real-valued symmetric bilinear form on $M$.

(ii) The positive set $M^{+}$is the set of $q \in M$ such that $w^{T} \tilde{B} w>0$ holds for $w \neq 0$.

(ii) The negative set $M^{-}$is the set of $q \in M$ such that $w^{T} \tilde{B} w<0$ holds for $w \neq 0$.

(iii) The indefinite set $M^{+/-}$is the set of $q \in M$ such that $w^{T} \tilde{B} w$ may take positive, negative and zero value for $w \neq 0$.

(iv) The degenerate set $M^{\emptyset}$ is the set of $q \in M$ such that $w^{T} \tilde{B} w=0$ holds for all $w$.

\section{Control Results}

The following section contains the main results of this paper. Our goal is to determine conditions that can be expressed in terms of properties of a real-valued symmetric bilinear form.

Theorem 12: Let $\Sigma=\{M, \mathbb{G}, V=0, \mathscr{Y}, U\}$ be a simple mechanical system underactuated by an arbitrary number of controls. If $\operatorname{Sym}^{\infty}(\mathscr{Y}) \neq T M$ then $\Sigma$ cannot be driven to rest from an arbitrary initial configuration and velocity.

Proof: It follows from (5) that if $\operatorname{Sym}^{\infty}(\mathscr{Y}) \neq T M$ then either $\frac{d s^{b}}{d t}=0$ for all $b$ or there exists at least one $\frac{d s^{b}}{d t} \neq 0$ and is linear in $s$ and $w$. Clearly, as $s$ approaches zero so does $\frac{d s^{b}}{d t}$. Therefore, $\Sigma$ can never reach $\operatorname{Sym}^{\infty}(\mathscr{Y})$ which contains rest.

It follows from (1) that if we choose $u^{a}$ sufficiently large, $w^{a}$ can achieve any value for each $a=1, \ldots, m$. We need to show that it is possible to do this with an arbitrarily small influence on the configuration $q$ and orthogonal velocity component $s$.

Lemma 13: Let us assume that the set of control inputs $u^{a}(t)$ be continuous over the interval $(t, t+\delta)$. Then given any $\varepsilon>0$ and $\delta>0$ there exists a constant $M$ such that $u^{a}<M, w^{a}$ can achieve any value in time $\delta$ such that $\mid q(t+$ $\delta)-q(t) \mid<\varepsilon$ and $|s(t+\delta)-s(t)|<\varepsilon$ for each $a=1, \ldots, m$.

Proof: If $u^{a}(t)$ is continuous on the open interval $(t, t+$ $\delta)$ then it follows from (1) that $\frac{d w}{d t}(t)$ is continuous over the same interval. This implies that $w(t), s(t)$ and $q(t)$ are continuous on the closed interval $[t, t+\delta]$. Then by the MeanValue Theorem for vector-valued functions we conclude that the rate of change of $s(t)$ and $q(t)$ achieves a bounded value on the open interval between $t$ and $t+\delta$. Since $s(t)$ and $q(t)$ change in a bounded fashion over $(t, t+\delta)$ then we can always find a sufficiently large $M$ such that $u^{a}<M$ and $w^{a}$ achieves any value for all $a=1, \ldots, m$.

Lemma 14: Let $\Sigma=\{M, \mathbb{G}, V, \mathscr{Y}, U\}$ be a simple mechanical system underactuated by one control. If $\dot{\gamma}\left(t_{0}\right) \in \mathscr{Y}_{\gamma\left(t_{0}\right)}$ then $\Sigma$ is $\varepsilon$-stabilizable to rest from an arbitrary configuration.

Proof: This is an application of Lemma 13.

Now we state our main result.

Theorem 15: Let $\Sigma=\{M, \mathbb{G}, V, \mathscr{Y}, U\}$ be a simple mechanical system underactuated by one control. If the Lie derivative of the $\operatorname{det}(\tilde{B}(q))$ with respect to $Y_{a}$ for some $a=$ $1, \ldots, m$ is nonzero for all $q \in \operatorname{cl}\left(M^{+}\right)$and $q \in \operatorname{cl}\left(M^{-}\right)$then $\Sigma$ is $\varepsilon$-stabilizable to rest from an arbitrary initial configuration and velocity.

Proof: Given Lemma 14, it is sufficient to show that $\Sigma$ can be driven to the affine leaf $\mathscr{A}_{0}=\mathscr{Y}$ given an arbitrary initial configuration $q_{0}$ and velocity $\left(w_{0}, s_{0}\right)$. It follows from Lemma 13 that there exists a set of bounded inputs $u^{a}$ such that $w^{a}$ can achieve any value consistent with $\tilde{B}$ and not leave the regions $M^{+}, M^{-}$, or $M^{+} /-$. Further, since $\frac{d s}{d t}$ depends on $w^{a}$ in a quadratic fashion whereas the configuration $q$ depends on $w^{a}$ linearly then it is also true that there exists a set of bounded inputs $u^{a}$ such that given a configuration that lies in the appropriate region, $s$ can be driven to rest without leaving that region.

The basic idea for the remainder of the proof is that $M$ can be partitioned into regions $M^{+}, M^{-}, M^{+/-}$, and $M^{\emptyset}$. Let us 
examine the six possible cases. ${ }^{(i)}$ If the initial configuration $q_{0} \in M^{+/-}$then there exists a set of inputs $u$ to drive $w$ so that $w^{T} B w<0$ or $w^{T} B w>0$ and $\frac{d s}{d t}<0$ or $\frac{d s}{d t}>0$. This implies that $s_{0}$ can always be decreased or increased and thus driven to $s=0$ from above or below. ${ }^{(i i)}$ Let $q_{0} \in M^{+}$and $s_{0}<0$. Therefore, for all $q \in M^{+}$there exists a set of inputs $u$ to drive $w$ so that $w^{T} B w>0$ and $\frac{d s}{d t}>0$. This implies that $s_{0}$ can be increased and thus driven to $s=0$ from below. ${ }^{(i i i)}$ Let $q_{0} \in M^{-}$and $s_{0}>0$. Therefore, for all $q \in M^{-}$there exists a set of inputs $u$ to drive $w$ so that $w^{T} B w<0$ and $\frac{d s}{d t}<0$. This implies that $s_{0}$ can be decreased and thus driven to $s=0$ from above. ${ }^{(i v)}$ Let $q_{0} \in M^{+}$and $s_{0}>0$. If the closure $\operatorname{cl}\left(M^{+}\right)$is invariant and $\frac{d s}{d t}>0$ for all $q \in M^{+}$then $\Sigma$ cannot be driven to $s=0$. Let us assume that $L_{Y_{a}}(\operatorname{det}(\tilde{B}(q))) \neq 0$ for $q \in \operatorname{cl}\left(M^{+}\right)$and some $a=1, \ldots, m$. This implies that there exists a set of inputs $u$ to drive $q$ to either $M^{-}$or $M^{+/-}$. The proof is now a consequence of (iii) or $(i)$, respectively. ${ }^{(v)}$ Let $q_{0} \in M^{-}$and $s_{0}<0$. If the closure $\operatorname{cl}\left(M^{-}\right)$is invariant and $\frac{d s}{d t}<0$ for all $q \in M^{-}$then $\Sigma$ cannot be driven to $s=0$. Let us assume that $L_{Y_{a}}(\operatorname{det}(\tilde{B}(q))) \neq 0$ for $q \in \operatorname{cl}\left(M^{-}\right)$and some $a=1, \ldots, m$. This implies that there exists a set of inputs $u$ to drive $q$ to either $M^{+}$or $M^{+/-}$. The proof is now a consequence of $(i i)$ or $(i)$, respectively. ${ }^{(v i)}$ Finally, let us assume that $q \in M^{\text {emptyset }}$. The boundary of $M^{+}$and $M^{-}$is $M^{\text {emptyset }}$. If we assume that $L_{Y_{a}}(\operatorname{det}(B(q))) \neq 0$ for $q \in \operatorname{cl}\left(M^{+}\right)$and $q \in \operatorname{cl}\left(M^{-}\right)$then $L_{Y_{a}}(\operatorname{det}(B(q))) \neq 0$ for all $q \in M^{\emptyset}$. This implies there exists a there exists a set of inputs $u$ to drive $q$ to either $M^{-}, M^{+}$or $M^{+/-}$. The proof is now a consequence of $(i),(i i)$ or (iii), respectively.

\section{APPLICATION}

\section{A. Control Analysis}

This section contains the application of the preceding theory to the forced planar rigid body (FPRB). The example is simple and intended to illustrate our theory. Let us simplify our calculations by assuming $m=1, J=1$ and $h=1$. We begin by constructing the control vector fields given $\left\{F^{1}, F^{2}, F^{3}\right\}$ :

$$
\begin{aligned}
& Y_{1}=\cos \theta \frac{\partial}{\partial x}+\sin \theta \frac{\partial}{\partial y} \\
& Y_{2}=-\sin \theta \frac{\partial}{\partial x}+\cos \theta \frac{\partial}{\partial y}-\frac{\partial}{\partial \theta}, \\
& Y_{3}=\frac{\partial}{\partial \theta} .
\end{aligned}
$$

Let us consider the case when the input distribution is given by $\mathscr{Y}_{12}=\left\{Y_{1}, Y_{2}\right\}$. We construct the single element in the orthonormal set $\mathscr{Y}^{\perp}=\left\{Y^{\perp}\right\}$ given $\mathscr{Y}_{12}$ by inspection:

$$
Y^{\perp}=\frac{-\sin \theta}{\sqrt{2}} \frac{\partial}{\partial x}+\frac{\cos \theta}{\sqrt{2}} \frac{\partial}{\partial y}+\frac{1}{\sqrt{2}} \frac{\partial}{\partial \theta} .
$$

Next, we compute the coefficients of the intrinsic realvalued symmetric bilinear form $B$. The only non-zero coef- ficients are the off-diagonal terms

$$
\begin{aligned}
& B_{12}=-\frac{1}{2}\left\langle\left\langle\left\langle Y_{1}: Y_{2}\right\rangle, Y^{\perp}\right\rangle\right\rangle=\frac{\sqrt{2}}{4}, \\
& B_{21}=-\frac{1}{2}\left\langle\left\langle\left\langle Y_{2}: Y_{1}\right\rangle, Y^{\perp}\right\rangle\right\rangle=\frac{\sqrt{2}}{4},
\end{aligned}
$$

Now we expand the expression $w^{T} B w$ to get

$$
B_{i j} w^{i}(t) w^{j}(t)=\frac{\sqrt{2}}{2} w^{1}(t) w^{2}(t)
$$

Equation (7) is a bivariate quadratic function in $w^{1}$ and $w^{2}$. The graph of this function is a saddle. This follows from the second derivative test:

$$
\left(\frac{\partial^{2} Q_{12}}{\partial w^{1} \partial w^{1}}\right)\left(\frac{\partial^{2} Q_{12}}{\partial w^{2} \partial w^{2}}\right)-\left(\frac{\partial^{2} Q_{12}}{\partial w^{1} \partial w^{2}}\right)^{2}=-\frac{1}{2} .
$$

Following a similar procedure, we calculate the intrinsic real-valued symmetric bilinear forms associated with the input distributions $\mathscr{Y}_{13}=\left\{Y_{1}, Y_{3}\right\}$ and $\mathscr{Y}_{23}=\left\{Y_{2}, Y_{3}\right\}$ which are shown in Table I.

TABLE I

BIVARIATE QUADRATIC EQUATIONS

\begin{tabular}{|c|c|}
\hline Input Distribution & $B_{i j} w^{i} w^{j}$ \\
\hline \hline $\mathscr{Y}_{13}$ & $-w^{1}(t) w^{3}(t)$ \\
\hline $\mathscr{Y}_{23}$ & $-w^{2}(t) w^{2}(t)+w^{2}(t) w^{3}(t)$ \\
\hline
\end{tabular}

Again, the graph of the bivariate quadratics associated with $\mathscr{Y}_{13}$ and $\mathscr{Y}_{23}$ are saddles. It then follows from Theorem 15 that FPRB given the input distributions $\mathscr{Y}_{12}, \mathscr{Y}_{13}$, and $\mathscr{Y}_{23}$ is $\varepsilon$-STR from an arbitrary initial configuration and velocity.

Now we analyze a forced planar rigid body with a single control force. Let us consider the two cases when the input distribution is given by $\mathscr{Y}_{1}=\left\{Y_{1}\right\}$ and $\mathscr{Y}_{3}=\left\{Y_{3}\right\}$. We observe that in each case the input distribution is geodesically invariant. It then follows from Theorem 12 that FBRB given the input distributions $\mathscr{Y}_{1}$ and $\mathscr{Y} / 3$ cannot be driven to rest from an arbitrary initial configuration and velocity.

Finally, let us consider the case when $\mathscr{Y}_{2}=\left\{Y_{2}\right\}$. We check for geodesic invariance by computing the following symmetric products

$$
\begin{aligned}
\left\langle Y_{2}: Y_{2}\right\rangle & =\cos \theta \frac{\partial}{\partial x}+\sin \theta \frac{\partial}{\partial y} \\
\left\langle Y_{2}:\left\langle Y_{2}: Y_{2}\right\rangle\right. & =\sin \theta \frac{\partial}{\partial x}-\cos \theta \frac{\partial}{\partial y} .
\end{aligned}
$$

We observe that the $\operatorname{span}\left\{Y_{2},\left\langle Y_{2}: Y_{2}\right\rangle,\left\langle Y_{2}:\left\langle Y_{2}: Y_{2}\right\rangle\right\}=\right.$ $T M$. Since the $\operatorname{Sym}^{\infty}\left(\mathscr{Y}_{2}\right)=T M$ and the system is underactuated by two controls, our theorems are not applicable. However, we are able to conclude the following weaker result.

We begin by constructing the two elements of the orthonormal set $\mathscr{Y}^{\perp}$ by inspection:

$$
\begin{aligned}
& Y_{1}^{\perp}=-\cos \theta \frac{\partial}{\partial x}+-\sin \theta \frac{\partial}{\partial y} \\
& Y_{2}^{\perp}=-\frac{\sin \theta}{\sqrt{2}} \frac{\partial}{\partial x}+\frac{\cos \theta}{\sqrt{2}} \frac{\partial}{\partial y}+\frac{1}{\sqrt{2}} \frac{\partial}{\partial \theta} .
\end{aligned}
$$


Now we construct the quadratics:

$$
\begin{aligned}
\frac{d s^{1}}{d t} & =-\frac{1}{2} s^{2}(t) s^{2}(t)+w^{2}(t) w^{2}(t), \\
\frac{d s^{2}}{d t} & =\frac{1}{2} s^{1}(t) s^{2}(t)-\frac{\sqrt{2}}{2} w^{2}(t) s^{1}(t) .
\end{aligned}
$$

It follows from (8) and (9) that it is not possible to simultaneously increase $s^{1}(t)$ and $s^{2}(t)$ nor is it possible to simultaneously decrease $s^{1}(t)$ and $s^{2}(t)$. We observe that the threshold for increasing or decreasing $s^{1}(t)$ and $s^{2}(t)$ occurs at

$$
w^{2}(t)=\frac{1}{\sqrt{2}} s^{2}(t)
$$

This implies the weaker result that FPRB given the input distribution $\mathscr{Y}_{2}$ cannot be driven directly to rest from above or below the distribution $\mathscr{Y}$.

\section{B. Nonlinear Control Law}

The computable sufficient conditions associated with Theorem 15 naturally gives rise to a nonlinear control law for simple mechanical systems underactuated by one control and where $M=M^{+/-}$. The nonlinear control law consists of three separate temporal stages. We fix the control input over each stage or time interval. Here are the objectives for each interval.

1) Drive $w^{a}(t)$ to a control velocity $w_{c}^{a}(t)$ for all $a=$ $1, \ldots, m$ necessary for achieving a desired rate of change $\frac{d s}{d t}$.

2) Fix $w_{c}^{a}(t)$ and drive $s(t)$ to zero.

3) Drive $w_{c}^{a}(t)$ to zero for all $a=1, \ldots, m$.

Let us consider FPRB when the input distribution is given by $\mathscr{Y}_{13}=\left\{Y_{1}, Y_{3}\right\}$. Our nonlinear control law is illustrated in Figure 2 and 3 given the initial velocity values $\left\{w^{1}(0)=-10, w^{2}(0)=20, s(0)=10\right\}$ and $\left\{w^{1}(0)=\right.$ $\left.10, w^{2}(0)=20, s(0)=-30\right\}$, respectively.

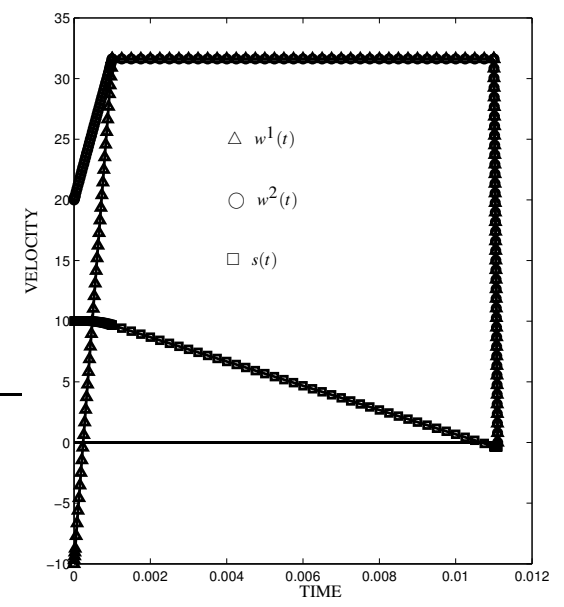

Fig. 2. Plot of velocity components vs. time given $s(0)=10$

Note that the temporal length of each stage and the distance the velocity component $s$ is from zero at the end of the final stage can be reduced by choosing a larger input over each stage.

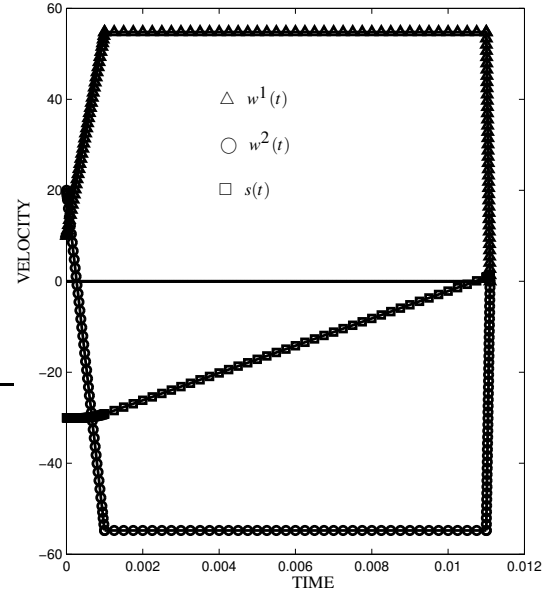

Fig. 3. Plot of velocity components vs. time given $s(0)=-30$

\section{Future WORK}

Simple mechanical systems underactuated by one control is a special case and is the simplest case next to fully actuated systems which can always be driven to rest. Future research will focus on characterizing coordinate invariant properties of the intrinsic vector-valued symmetric bilinear form that allow motion in the input foliation.

\section{REFERENCES}

[1] F.Bullo and A.Lewis. Low-order controllablility and kinematic reductions for affine connection control systems. SIAM Journal on Control and Optimization 44(3), pages 885-908, 2005.

[2] F. Bullo and M. Zefran. Modeling and controllability for a class of hybrid mechanical systems, IEEE Transactions on Robotics and Automation 18(4):563-573, 2002.

[3] H.J. Sussmann. A general theorem on local controllability. SIAM Journal of Control and Optimization, 25(1):158-194, 1987.

[4] A. Lewis and R. Murray. Configuration controllability of simple mechanical control systems. SIAM Journal on Control and Optimization, 35(3):766-790,1997.

[5] R. Hirschorn and A. Lewis. Geometric local controllability: secondorder conditions. Proceedings of the 41st IEEE Conference on Decision and Control, 1:368-369, December 2002.

[6] F. Bullo and A. Lewis. Geometric Control of Mechanical Systems. Springer Science+Business Media, New York, NY, 2005.

[7] A.Kelly and R.Murray. Geometric phases and robotic locomotion. J. Robotic Systems, 12(6):417-431, 1995.

[8] J. Cortes, S. Martinez, J.P. Ostrowski, and H. Zhang. Simple mechanical control systems with constraints and symmetry. SIAM Journal on Control and Optimization, 41(3):851-874,2002.

[9] J.E.Colgate and K.Lynch. Mechanics and control of swimming: a review. IEEE J. Oceanic Engineering, 29(3):660-673, 2004.

[10] J. Cortes, S. Martinez and F. Bullo. On nonlinear controllability and series expansions for Lagrangian systems with dissipative forces. IEEE Transactions on Automatic Control, 47(8):1396-1401, 2002.

[11] A. Lewis Simple mechanical control systems with constraints. IEEE Transactions on Automatic Control, 45(8):1420-1436, August 2000.

[12] M.P. do Carmo. Riemannian Geometry. Birkhauser, Boston, MA, English edition, 1992.

[13] R. Abraham and J. Marsden. Foundations of Mechanics. The Benjamin-Cummings Publishing Company, Reading, MA,second edition, 1978.

[14] A. Lewis. Affine connections and distributions with applications to nonholonomic mechanics. Reports on Mathematical Physics, 42(1):135-164,1998. 